\title{
MicroRNA-140-5p Inhibits the Progression of Colorectal Cancer by Targeting VEGFA
}

\author{
Wenbo Zhang ${ }^{\mathrm{a}}$ Chen Zou ${ }^{\mathrm{a}}$ Lei Pan ${ }^{\mathrm{a}}$ Ying Xu ${ }^{\mathrm{b}}$ Weidong Qia Gui Ma ${ }^{\mathrm{a}}$ \\ Yongzhong $\mathrm{Hou}^{\mathrm{c}}$ Pengcheng Jiang ${ }^{\mathrm{a}}$ \\ aDepartment of General Surgery, Affiliated People's Hospital of Jiangsu University, Zhenjiang, \\ 'Laboratory Center, Affiliated People's Hospital of Jiangsu University, Zhenjiang, 'Institute of Life \\ Sciences, Jiangsu University, Zhenjiang, China
}

\section{Key Words}

MiR-140-5p • Colorectal cancer • VEGFA

\begin{abstract}
Background: microRNAs (miRNAs) are small non-coding RNAs and have been shown to play a crucial role in the colorectal cancer (CRC) tumorigenesis and progression. The aim of this study was to investigate the clinical significance and prognostic value of miR-140-5p in CRC. The exact functions and the underlying molecular mechanisms of miR-140-5p in CRC was further determined. Methods: miR-140-5 $p$ expression was detected in CRC samples, their adjacent nontumor tissues as well as CRC cell lines by RT-qPCR. Cell proliferation was detected using CCK-8, and cell invasion and migration were evaluated using Transwell assay. The direct regulation of VEGFA by miR-140-5p was identified using luciferase reporter assay. Results: miR-140-5p was significantly dowregulated in CRC tissues and cell lines. Downregulation of miR-140-5p was significantly correlated with advanced CRC stage and poorer overall survival. Both gain-of-function and loss of function studies demonstrated that miR-140-5p acted as a tumor suppressor by inhibiting cell proliferation, migration and invasion. Integrated analysis identified VEGFA as a direct and functional target gene of miR-140-5p. Silencing VEGFA by small interfering RNA (siRNA) resembled the phenotype resulting from ectopic miR-140$5 p$ expression, while overexpression of VEGFA attenuated the effect of miR-140-5p on CRC cells. Conclusions: Our results suggested a tumor suppressive role of miR-140-5p in CRC tumorigenesis and progression by targeting VEGFA.
\end{abstract}

Copyright @ 2015 S. Karger AG, Basel

\section{Introduction}

Colorectal cancer (CRC) is the third most common cancer in men and the second in women worldwide [1]. Although substantial progress has been made in the past decades, including surgical treatment, radiotherapy and chemotherapy, patients with advanced CRC continue to experience poor prognosis and a high death rate [2]. Consequently, a better understanding of the molecular mechanisms underlying CRC development and progression is urgently needed.

W. Zhang, C. Zou, L. Pan contributed equally to this work. 


\section{Cellular Physiology Cell Physiol Biochem 2015;37:1123-1133

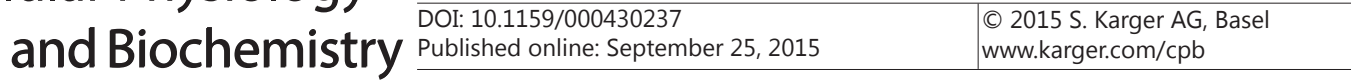 \\ Zhang et al.: miR-140-5p in CRC}

MicroRNAs (miRNAs) are a family of small non-coding 18-25 nucleotides RNA, which function in the post-transcriptional regulation of gene expression by targeting mRNA for cleavage or translational repression [3]. Mechanistically, the 3' untranslated region (UTR) of mRNA is targeted through a semi-complimentary seed sequence (6-9 bp) in miRNAs, which guides binding to the miRNA response elements. miRNAs number approximately 1000 , accounting for $2 \%-5 \%$ of the whole human genome, and regulate the expression of at least $20 \%$ of human genes [4]. A single miRNA can manipulate multiple target gene expressions, initiate signaling pathways and provoke signal crosstalk, and vice versa. Therefore, miRNAs may play a crucial role in epigenetic regulation of fundamental cellular processes, such as cell proliferation, apoptosis, differentiation, and migration, which strongly indicates that they may function as potential oncogenes or tumor suppressors in cancer development [5, 6]. Indeed, deregulations of a few miRNAs have been proved in the CRC tumorigenesis in previous studies. Of those most widely investigated miRNAs, some are classified as oncogenes in CRC, such as miR-21, miR-155, miR-31 and miR-92a, while others are considered as tumor suppressors represented by Let-7, miR-143, miR-145 and miR-215 [7, 8].

miR-140-5p has not attracted much attention until more recently. It is encoded within intron 16 of $W w p 2$, an E3 ubiquitin ligase on chromosome 16 [9]. Expression profiling of tumors and normal tissues has revealed a possible tumor suppressive role for miR-140-5p in many cancers, including ovarian cancer [10], lung cacner [11,12], CRC [13, 14], osteosarcoma [13], hepatocellular carcinoma [15], breast cancer [16], esophageal cancer [17] and basal cell carcinoma [18]. By targeting various targets, miR-140-5p has shown to critically involve in tumor cell proliferation, apoptosis, vascularization, migration and invasion. These previously confirmed targets includes Sp1 [9], IGF1R [11], MMD [12], HDAC4 [13], Smad2 [14], TGFBR1 [15], FGF9 [15], SOX2 [16], Slug [17], BMP2 [19], Smad3 [20], and ADAMTS5 [21]. Although miR-140-5p is generally accepted as a tumor suppressor, an oncogenic role of miR-140-5p has also been implicated depending on different cell context. For example, elevated levels of miR-140-5p are reported upon malignant progression of gliomas [22]. A decreased level of IGFBP-5, a miR-140-5p target [23], is related to disease recurrence in lung cancer [24] and to tamoxifen resistance in breast cancer [25].

With regard to the miR-140-5p in CRC tumorigenesis, there are still relatively few studies available and discrepancy was found between these studies. Zhai et al. demonstrated that overexpression of miR-140-5p abolished tumor formation and metastasis in colon cancer stem cells by directly targeting Smad2 [14]. A progressive loss of miR-140-5p expression was also revealed from normal colorectal mucosa to primary tumor tissues, with further reduction in liver metastatic tissues. Higher miR-140-5p expression is significantly correlated with better survival in stage III and IV CRC patients. In contrast, Mosakhani and colleague found that miR-140-5p up-regulation were significantly associated with poorer overall survival in metastatic CRC patients with wild type KRAS/BRAF [26]. Song et al. showed that miR-140-5p is downregulated in CRC tissue compare with adjacent normal tissue, but is upregulated in colon cancer stem-like cells [13]. Its expression is related to chemoresistance, and blocking it partially sensitizes colon cancer stem-like cells that are resistant to 5-FU treatment. Based on these many controversial issues, the precise mechanism of miR-140-5p in CRC still need to be further investigated.

The aim of the present study was to demonstrate the role of miR-140-5p on CRC progression. We also explored its functions in cell proliferation, colony formation and apoptosis of CRC SW480 and HCT116 cells. Then the effects of ectopic miR-140-5p on the invasion and migration of CRC cells were determined. Finally, we identified the direct target of miR-140-5p in CRC cells.

\section{Materials and Methods}

Cell lines and patient tissue samples

Human CRC cell lines SW480, HT29, HCT116 cells were purchased from the cell bank of the Chines Academy of Sciences (Shanghai, China). NCM460 cell was purchased from American INCELL Corporation. 


\section{Cellular Physiology Cell Physiol Biochem 2015;37:1123-1133 \begin{tabular}{l|l|l}
\hline DOI: 10.1159/000430237 & C 2015 S. Karger AG, Basel
\end{tabular} and Biochemistry Published online: September 25, 2015 \\ Zhang et al.: miR-140-5p in CRC}

All cell lines were cultured in DMEM (Invitrogen, Carlsbad, CA) supplemented with 10\% fetal bovine serum (Invitrogen), $100 \mathrm{IU} / \mathrm{mL}$ penicillin, and $100 \mu \mathrm{g} / \mathrm{mL}$ streptomycin in a humid atmosphere containing 5\% $\mathrm{CO}_{2}$ at $37^{\circ} \mathrm{C}$.

Thirty six pairs of CRC tissues and matched adjacent normal tissues were obtained from patients in Affiliated People's Hospital of Jiangsu University, with written informed consent and agreement. The tissues were snap-frozen in liquid nitrogen and stored at $-80^{\circ} \mathrm{C}$ until RNA extraction. This study was approved by the Medical Ethics Committee of Affiliated People's Hospital of Jiangsu University (Registration number: SQ20150024).

\section{RNA extraction and RT- $q P C R$}

Total RNA was isolated according to the manufacturer's Instructions by using Trizol (Invitrogen) and treated with RNase-free DNase I (Qiagen, Hilden, Germany). Expression of mature miR-140-5p was assayed using stem-loop RT (Fermentas, Glen Burnie, USA) followed by real-time PCR analysis (TakaRa, Dalian, China) according to the manufacturer's instructions. U6 snRNA was used as internal standard to normalize the expression of miR-140-5p. Expression levels of VEGFA mRNA was normalized to $\beta$-actin mRNA. The relative expression levels of each sample were measure using the $2^{-\Delta \Delta C T}$ method. The specific primer pairs used in this study are shown in Table 1.

\section{microRNA and siRNA transfection assay}

The miR-140-5p mimic, normal mimic control, miR-140-5p inhibitor and negative inhibitor control were chemically synthesized by Shanghai GenePharma Company (Shanghai, China). VEGFA siRNA and its scramble siRNA control were also purchased from GenePharma Company. The sequence for VEGFA siRNA and scramble siRNA were as follows: VEGFA siRNA, sense: 5'-GGCAGAAUCAUCACGAAGUTT-3', antisense: 5'-ACUUCGUGAUGAUUCUGCCTT-3'; scramble siRNA, sense: 5'-UUCUCCGAACGUGUCACGUTT-3', antisense: 5'-ACGUGACACGUUCGGAGAATT-3'. CRC Cells were plated in 6-well plates and transfected with 100nM of the mimic, mimic control, inhibitor, inhibitor control using Lipofectamine 2000 (Invitrogen) according to the manufacture's protocol. VEGFA siRNA or scramble siRNA were transfected using the same approach.

\section{Cell proliferation, colony formation assays, apoptosis}

Cells $\left(2 \times 10^{3}\right)$ were seeded into 96-well plates, and the proliferation of the cells was assayed at 0,24 , 48,72 and $96 \mathrm{~h}$ using a CCK-8 kit (Dojindo Laboratories, Kumamoto, Japan) according to the manufacturer's instructions. Cell variability was assessed by measurement of absorbance at $450 \mathrm{~nm}$ using a microplate reader. For colony formation assay, cells (200/well) were plated into six-well plates and cultured for 14 days. At the end of the culture period, the cells were stained with $0.05 \%$ crystal violet for at least 1 hour. The number of colonies were assessed in four different fields. Cell apoptosis assays were performed using an Annexin V-FITC/PI Apoptosis Detection Kit (BD Bioseciences). $1 \times 10^{4}$ cells were stained according to the manufacturer's protocol and then analyzed with a flow cytometry (BD Biosciences) equipped with a CellQuest software (BD Biosciences).

\section{Wound healing and Matrigel invasion assays}

For the wound healing assay, cells $\left(5 \times 10^{5}\right)$ were seeded into six-well plates and cultured under standard conditions. When the cells reached confluence, a wound was made by scraping the cell monolayer

Table 1. Real-time PCR primers

\begin{tabular}{lll}
\hline Gene & \multicolumn{2}{c}{ Sequence $\left(5^{\prime}\right.$-3') } \\
\hline miR-140-5p & RT & GTCGTATCCAGTGCAGGGTCCGAGGTATTCGCACTGGATACGACCTACCATA \\
& Forward & GAGTGTCAGTGGTTTTACCCT \\
& Reverse & GCAGGGTCCGAGGTATTC \\
U6 & Forward & CTCGCTTCGGCAGCACA \\
& Reverse & AACGCTTCACGAATTTGCGT \\
VEGFA & Forward & GGCAGAATCATCACGAAGT \\
& Reverse & CACAGGATGGCTTGAAGAT \\
\multirow{2}{*}{-actin } & Forward & AGTGTGACGTGGACATCCGCAAAG \\
& Reverse & ATCCACATCTGCTGGAAGGTGGAC \\
\hline
\end{tabular}




\section{Cellular Physiology Cell Physiol Biochem 2015;37:1123-1133 \begin{tabular}{l|l|l}
\hline DOI: 10.1159/000430237 & (C) 2015 S. Karger AG, Basel
\end{tabular} and Biochemistry Published online: September 25, 2015 \\ Zhang et al.: miR-140-5p in CRC}

with a $200 \mu$ l pipette tip. Cell migration was determined by measuring the movement of cells into the scraped area. The process of wound closure was observed and photographed after $24 \mathrm{~h}$. For the cell invasion assay, $1 \times 10^{5}$ cells in serum-free medium were added into the upper chamber of an insert precoated with Matrigel (BD Bioscience). The lower chamber was filled with complete culture medium. After $48 \mathrm{~h}$ of incubation, the cells remaining on the upper surface of the membrane was removed, whereas the cells that had invaded through the membrane were stained with crystal violet. Photomicrographs of five regions were captured from duplicate chambers and the numbers of cells were counted.

Plasmid construction and luciferase reporter assays

The coding sequence of VEGFA was amplified using Pyrobest DNA polymerase (Fermentas) and then cloned into pcDNA3.1 (+) at the BamH I/Xho I stie to generate VEGFA expression vectors. The primers for VEGFA were as follows: F: 5'- TAC CGA GCT CGG ATC CGC CAC CAT GAA CTT TCT GCT GTC TTG GGT GC-3', R: 5'-TAG ATG CAT GCT CGA GTC ACC GCC TCG GCT TGT CAC AT-3'. The putative target sites of the human VEGFA 3'UTR (WT) segments for miR-140-5p were amplified with the following primers: F: 5'-CCG TGT AAT TCT AGA TGA CTG CTG TGG ACT TGA GTT GG-3', R: 5'-CGC CCC GAC TCT AGA ACT GTC ACC GAT CAG GGA GAG AG-3', and subcloned into PGL3 Basic Vector (Promega, Madison, WI, USA) at Xba I site. Site-directed mutagenesis of the miR-140-5p seed sequence in the VEGFA 3'-UTR (Mut-VEGFA) was performed using the QuickChange TM Site-Directed Mutagenesis Kit (Stratagene, La Jolla, CA, USA). For luciferase assays, the reporter plasmid was cotransfected with a control Renilla luciferase vector into SW480 and HCT116 cells in the presence of either miR-140-5p or mimic control. After 48h, cells were harvested, and the luciferase activity was measured using the Dual-Luciferase Reporter Assay System (Promega, Madison, WI, USA).

\section{Western blot analysis}

Cells were washed twice with ice-cold PBS and lysed in RIPA lysis buffer supplemented with protease inhibitors. Protein concentrations were determined using the BCA assay kit (Beyotime Inst Biotech, China). Protein samples were separated on SDS-PAGE and then electrotransferred to nitrocellulose membranes (Whatman, Maidstone, UK). Non-specific binding was blocked by $3 \%$ skimmed milk in Tris-buffered saline. Membranes were incubated overnight with the primary antibody: VEGFA and $\beta$-actin (Santa Cruz Bitechnology Inc., Santa Cruz, CA, USA). After washing and incubating with secondary antibodies, signals were visualized with Enhanced Chemiluminescence Plus Kit (GE Healthcare).

\section{Statistical analysis}

Data were expressed as the mean \pm SD from at least three independent experiments. The difference between groups was analyzed using Student t-test when comparing only two groups or one-way analysis of variance when comparing more than two groups. The correlation between miR-140-5p and VEGFA expression was evaluated using Spearman's correlation analysis. Kaplan-Meier method was used for overall survival curve analysis and the log-rank test was performed for statistical differences in subgroups. $P<0.05$ was considered statistically significant.

\section{Results}

Downregulated miR-140-5p expression in CRC tissues and cell lines

To study the expression and significance of miR-140-5p in CRC carcinogenesis, we measured the expression of miR-140-5p in 36 pairs of CRC tissues and their adjacent normal tissues. The results showed that miR-140-5p expression was significantly decreased in CRC tissues compared with their matched normal tissues (Fig. 1A). In addition, the correlation between miR-140-5p expression levels and clinicopathologic parameters was analyzed. The results revealed that the downregulation of miR-140-5p was significantly correlated with tumor stage and metastasis while no significant correlation was observed with regard to age, gender and tumor size (Fig. 1B, Table 2). To assess the prognostic relevance of miR140-5p in CRC, the cohort was dichotomized into miR-140-5p high and miR-140-5p low expressing tumors by using the median expression value of miR-140-5p as a cutoff point. The follow-up study showed that CRC patients with lower levels of miR-140-5p expression 


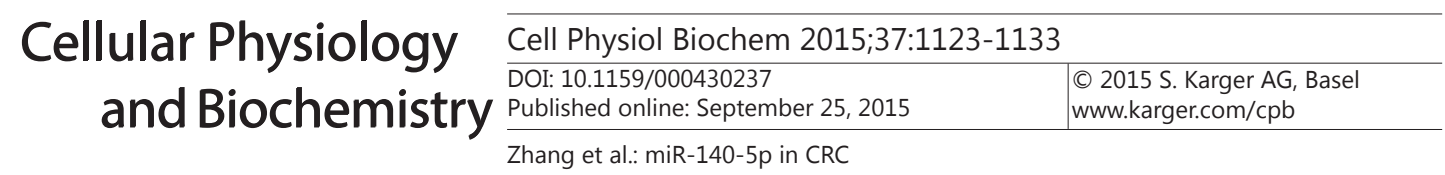

Table 2. Clinicopathological associations of miR-140-5p expression in primary CRC

\begin{tabular}{llll}
\hline Variable & Number of cases & Mean expression of miR-140-5p & $P$ value \\
\hline Age (years) & & & \\
$>60$ & 21 & $0.3590 \pm 0.0537$ & 0.546 \\
$\quad \leq 60$ & 15 & $0.3148 \pm 0.0412$ & \\
Gender & & & \\
$\quad$ Male & 22 & $0.2944 \pm 0.0386$ & 0.103 \\
$\quad$ Female & 14 & $0.4131 \pm 0.0653$ & \\
Tumor size (cm) & & & \\
$\geq 5$ & 21 & $0.3220 \pm 0.0425$ & 0.543 \\
$\quad<5$ & 15 & $0.3666 \pm 0.0620$ & \\
TNM stage & & & \\
Stage I/II & 20 & $0.4559 \pm 0.0436$ & \\
$\quad$ Stage III/IV & 16 & $0.1964 \pm 0.0330$ & \\
Lymph node status & & & \\
$\quad$ No metastasis & 20 & $0.4559 \pm 0.0436$ & \\
$\quad$ Metastasis & 16 & $0.1964 \pm 0.0330$ & \\
\hline
\end{tabular}

A

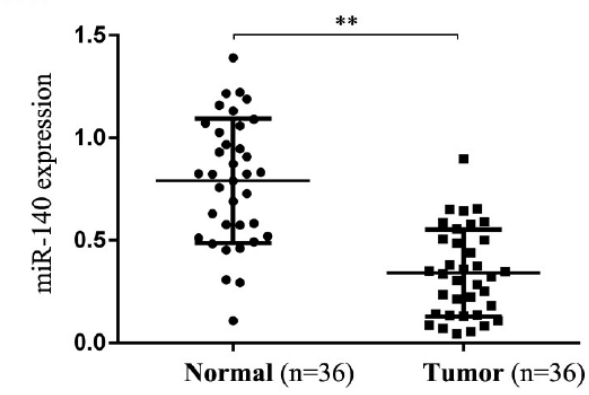

$\mathrm{C}$

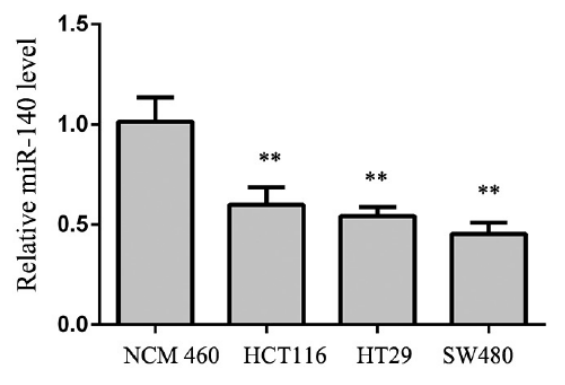

B

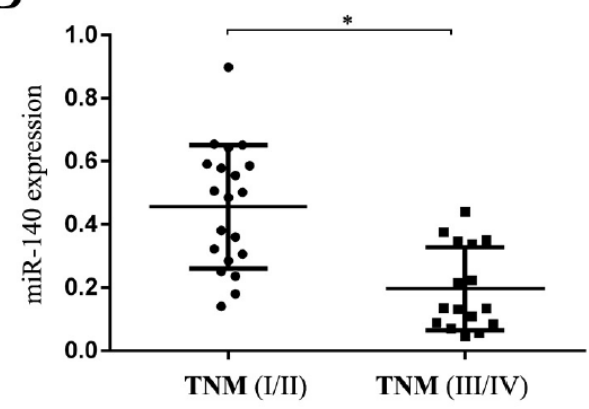

$\mathrm{D}$

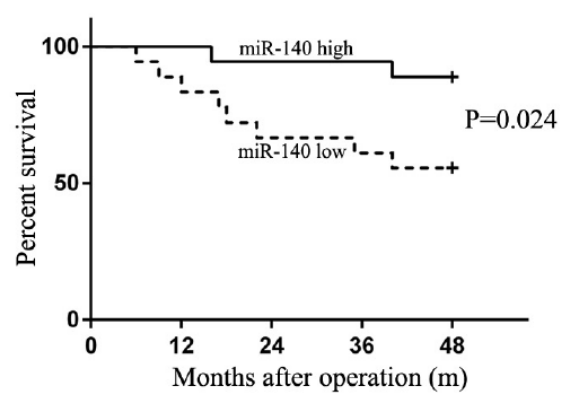

Fig. 1. Downregulation of miR-140-5p expression in CRC and its prognostic role. (A) the expression of miR140-5p in 36 pairs of CRC tissues and paired adjacent non-tumorous colorectal tissues were examined by RT-qPCR. (B) the expression level of miR-140-5p in TNM stages of CRC. (C) Kaplan-Meier curve for overall survival in 36 CRC patients according to miR-140-5p expression. (D) the expression levels of miR-140-5p in normal colorectal cell line (NCM460) and 3 CRC cell lines. Transcription levels were normalized to U6 expression. ${ }^{*} P<0.05,{ }^{* *} P<0.01$.

had a significantly poorer survival than those with higher levels of miR-140-5p expression (Fig. 1D). These results indicate that downregulation of miR-140-5p expression predicts poorer prognosis in CRC patients. We further assessed the expression levels of miR-140- 


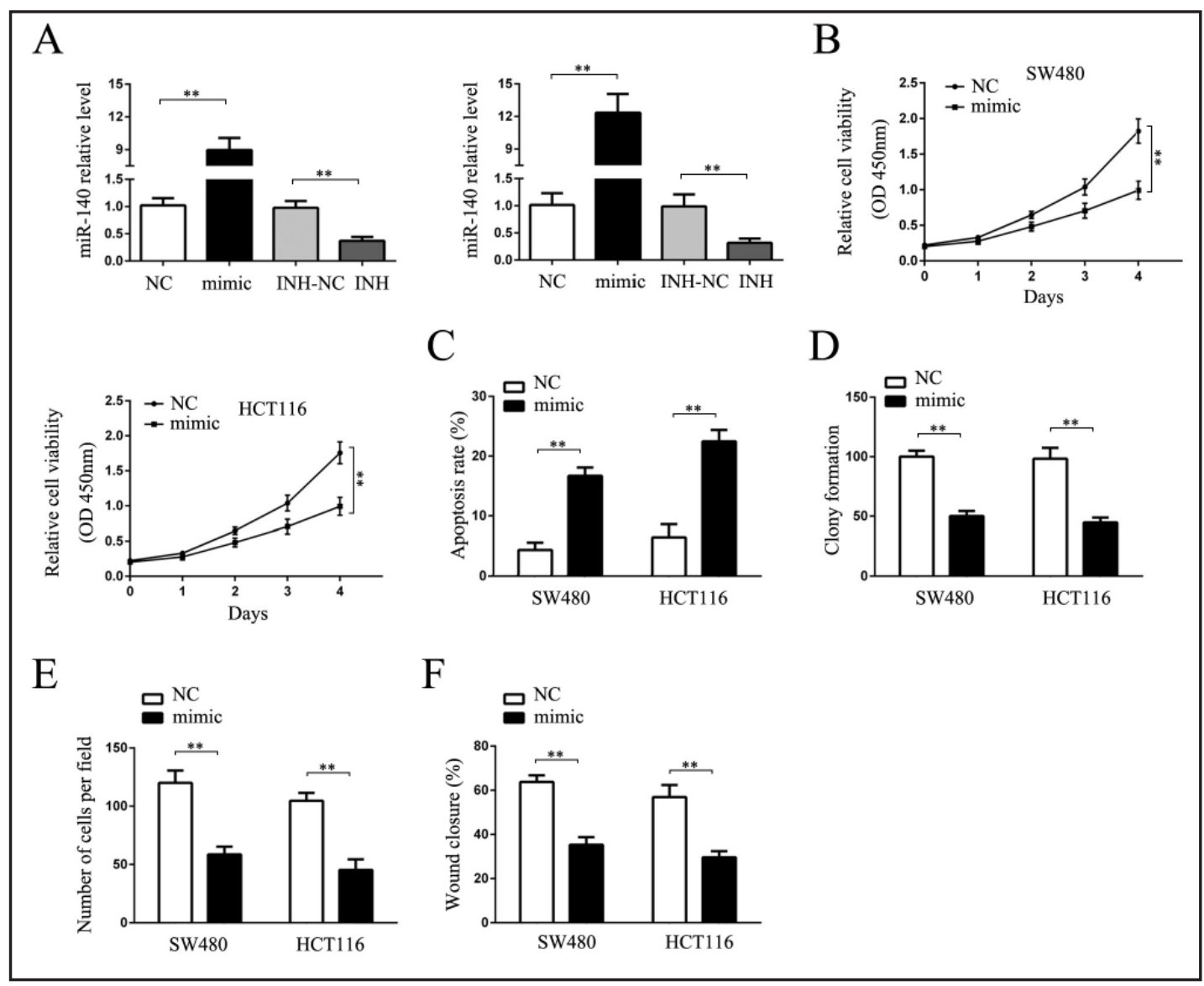

Fig. 2. Overexpression of miR-140-5p suppresses cell proliferation, induces cell apoptosis and inhibits cell migration and invasion of CRC cell lines in vitro. (A) relative expression levels of miR-140-5p in SW480 and HCT116 cells after transfection with miR-140-5p mimic, mimic control, inhibitor or inhibitor control. (B) cell viability assays (CCK-8). (C) cell apoptosis assays. (D) colony assays. (E) wound healing assays. (F) invasion assays. The invasion assays were determined using Transwell assays with Matrigel. ${ }^{* *} P<0.01$.

$5 p$ in different CRC cell lines (SW480, HCT116 and HT29). Consistent with the results found in CRC tissues, miR-140-5p levels were significantly lower in all three CRC cell lines tested compared with normal colorectal cell line (NCM460) (Fig. 1C). Taken together, these results suggest that the downregulation of miR-140-5p may play important roles in CRC carcinogenesis and progression.

Ectopic expression of miR-140-5p inhibits CRC cell proliferation and colony formation in vitro

To better understand the role of miR-140-5p in the development of CRC, we transfected SW480 and HCT116 cell lines with miR-140-5p mimic and miR-140-5p mimic control respectively. The transfection efficiency was validated by RT-qPCR (Fig. 2A). As shown in Fig. 2B, overexpression of miR-140-5p significantly suppressed cell proliferation of SW480 and HCT116 cells compared with their corresponding controls. miR-140-5p overexpression was also found to induce cell apoptosis in SW480 and HCT116 cells (Fig. 2C). Similarly, overexpression of miR-140-5p significantly decreased the ability of SW480 and HCT116 cells to form colonies (Fig. 2D). In contrast, knockdown of miR-140-5p using miR-140-5p inhibitor in both cell lines promoted cell growth and inhibited cell apoptosis (data were not shown). Taken together, these results demonstrate that miR-140-5p is able to regulate CRC cell growth. 
A

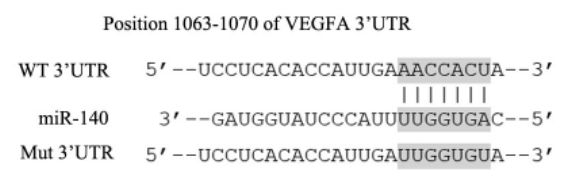

B

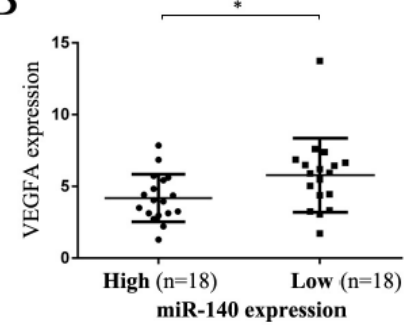

$\mathrm{C}$

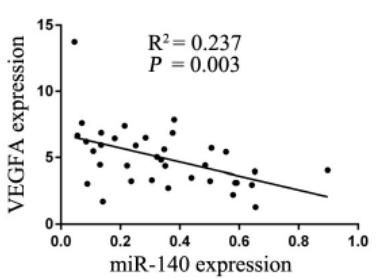

$\mathrm{E}$

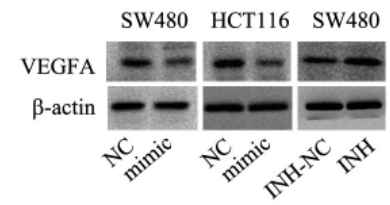

$\mathrm{D}$

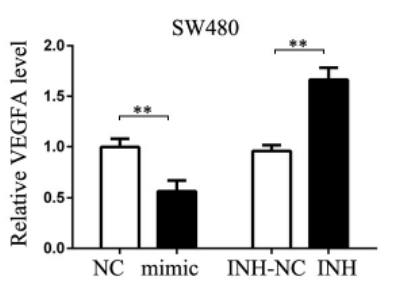

$\mathrm{F}$

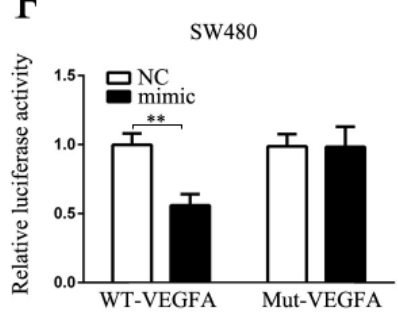

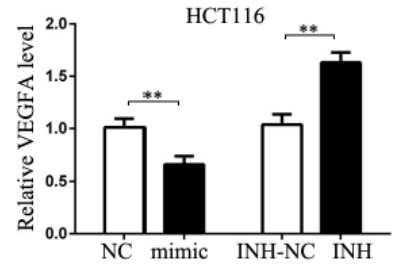

NC mimic INH-NC INH

Fig. 3. VEGFA is a direct target of miR-140-5p. (A) Predicted miR-140-5p target sequence in the 3'UTR of the VEGFA mRNA. Mutation was generated in the seed region of the VEGF 3'UTR as indicated. (B) the downregulation of miR-140-5p in CRC was associated with upregulation of miR-140-5p. (C) a statistically inverse correlation between miR-140-5p and VEGFA mRNA levels in CRC tissues by Spearman's correlation analysis. (D, E) the expression of VEGFA in SW480 and HCT116 cells transfected with miR-140-5p was measured by RT-qPCR and Western blot. (F) luciferase assay in SW480 and HCT116 cells cotransfected with miR-140-5p and a luciferase reporter containing the VEGFA 3'UTR (WT-VEGFA) or a mutant (Mut-VEGFA). Luciferase activities were measured $48 \mathrm{~h}$ post-transfection. ${ }^{*} P<0.05$, ${ }^{* *} P<0.01$.

\section{miR-140-5p inhibits CRC cell migration and invasion}

To further verify the oncogenic function of miR-140-5p in CRC, we next evaluated the effect of miR-140-5p on the migration and invasion capacity of CRC cells using the wound healing assay and Matrigel invasion assay. As shown in Fig. 2F, overexpression of miR-140-5p significantly suppressed tumor cell mobility in SW480 and HCT116 cells compared with their corresponding controls. Similarly, Transwell assays demonstrated that miR140-5p markedly decreased the invasive capacity of SW480 and HCT116 cells (Fig. 2E). In contrast, the wound healing and invasion of SW480 and HCT116 cells was increased when endogenous miR-140$5 p$ was silenced with miR-140-5p inhibitor (data were not shown). Therefore, these results suggest that miR-140-5p can suppress CRC cell migration and invasion in vitro.

miR-140-5p downregulates VEGFA by directly targeting its 3'UTR

To elucidate the molecular mechanisms by which miR-140-5p executes its function, we searched for the target genes of miR-140-5p using publicly available databases (Targetscan, miRANDA and miRWalk). In particular, we focused on VEGFA, which is well known as an oncogene. Upregulation of VEGFA is frequent in CRC and is associated with an increased ability of the malignancy to spread and with poorer prognosis $[27,28]$. Based on the putative target sites at 1063-1070 base pairs of the VEGFA 3'UTR (Fig. 3A), we supposed VEGFA 


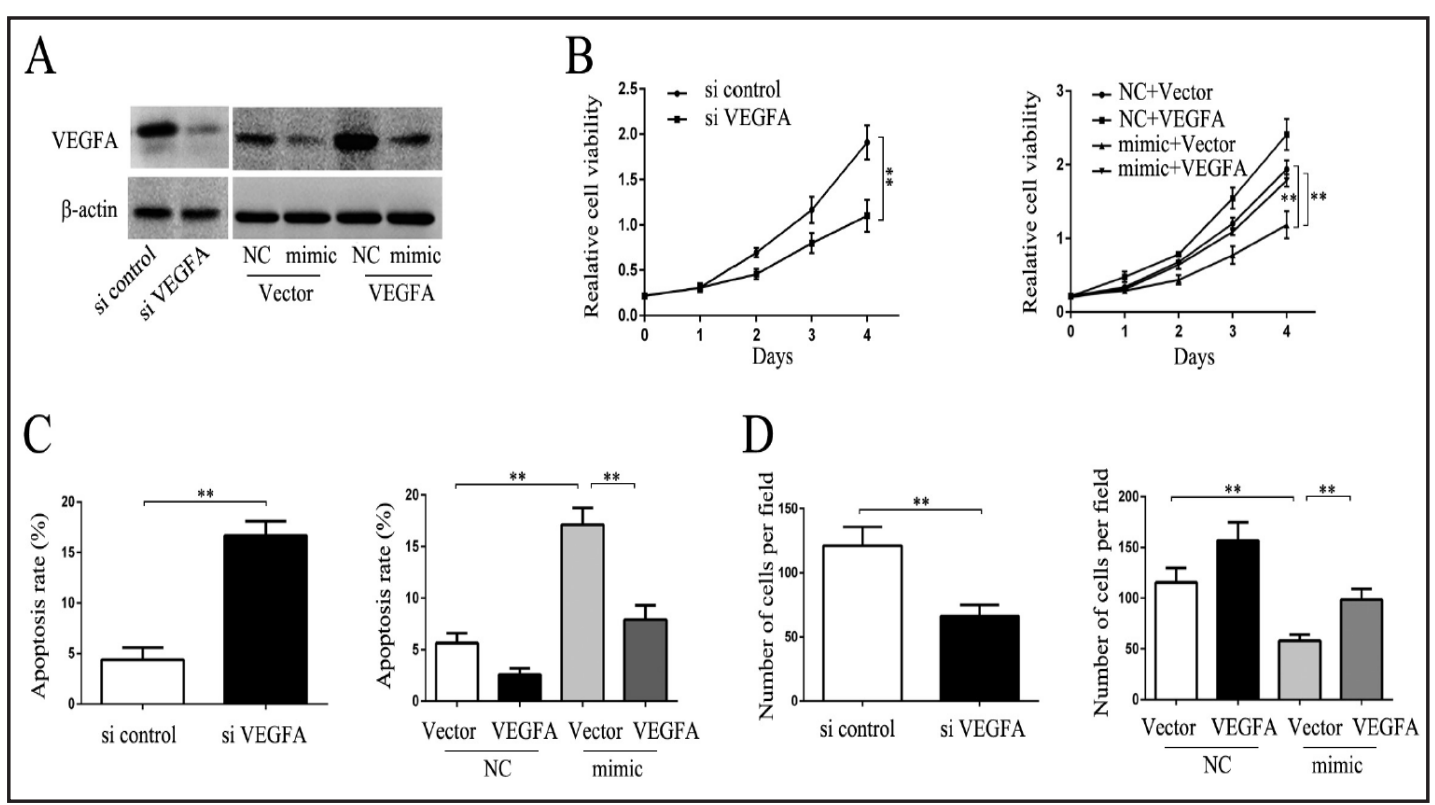

Fig. 4. VEGFA contributes to miR-140-5p-mediated growth and invasion inhibition in CRC cells. SW480 cells were transfected with specific siRNA targeting VEGFA, or VEGFA plasmid along with miR-140-5p. (A) Western blot analysis. (B) cell viability assays (CCK-8). (C) cell apoptosis assays. (D) Tanswell invasion assays. ** $P<0.01$.

was a potential downstream target of miR-140-5p. We found that the average expression level of VEGFA was significantly higher in CRC tissues than in matched normal tissues (Fig. 3B). In addition, a statistically significant inverse correlation was observed by Spearman's correlation analysis between expression levels of miR-140-5p and VEGFA mRNA (Fig. 3C). Furthermore, ectopic expression of miR-140-5p resulted in a significant reduction of VEGFA mRNA and protein expression, evaluated by RT-qPCR and western blot analysis (Fig. 3D and E). In contrast, inhibition of miR-140-5p led to the upregulation of VEGFA mRNA and protein. To validate whether VEGFA is the direct downstream target of miR-140-5p, we cloned dual-luciferase reporters containing the 3'UTR of VEGFA with seed sequence recognizing sites of wild type or mutant one (Fig. 3A). The miR-140-5p mimic or mimic control was cotransfected with plasmid containing 3'UTR of WT-VEGFA or MUT-VEGFA into SW480 and HCT116 cells. The results showed that upregulation of miR-140-5p significantly decreased the relative luciferase activity of WT-VEGFA-3'UTR in the both cells, but had no effect on the mutant of VEGFA-3'UTR (Fig. 3F). Taken together, these results suggest that miR-140-5p downregulates VEGFA expression by directly targeting its 3'UTR.

VEGFA is involved in miR-140-5p-mediated suppression of CRC cell growth and invasion

To investigate whether the regulatory effects of miR-140-5p on the proliferation, apoptosis and invasion of CRC cells are mediated by VEGFA, we applied siRNA-mediated VEGFA inhibition method to analyze whether it could replicated the tumor suppressor of miR-140-5p in CRC cell lines. siRNA-mediated suppression of VEGFA was confirmed by Western blot analysis (Fig. 4A). As Expected, VEGFA knockdown significantly inhibited cell growth and increased apoptosis in SW480 cells (Fig. 4B and C). Similarly, VEGFA knockdown suppressed cell invasion of SW480 cells (Fig. 4D). These results were similar to the effects of miR-140-5p overexpression. In consistent with that, reintroduction of VEGFA could rescue miR-140-5p-induced cell growth inhibition and apoptosis (Fig. 4B and C). The inhibitory effect of miR-140-5p on cell invasion could also be antagonized by VEGFA overexpression (Fig. 4D). Thus, these data suggest that VEGFA is a functional target of miR-140-5p. 


\section{Cellular Physiology Cell Physiol Biochem 2015;37:1123-1133 \begin{tabular}{ll|l} 
DOI: 10.1159/000430237 & $\begin{array}{l}\text { O 2015 S. Karger AG, Basel } \\
\text { www.karger.com/cpb }\end{array}$ \\
\hline
\end{tabular} \\ Zhang et al.: miR-140-5p in CRC}

\section{Discussion}

CRC has been described as a multistep disease due to the progressive accumulation of mutations and chromosomal rearrangements involving critical oncogenes or suppressors. Mounting studies have indicated that miRNAs can play crucial roles in epigenetic regulation of tumor-related gene expression and could act as an effective biomarker for the tumor diagnosis, prognosis or even therapy [5-8]. The aberrant expression of miR-140-5p is a frequent event in various kinds of cancers, suggesting an important role for miR-140-5p in the tumor initiation and progression. In the current study, we firstly demonstrated the clinical relevance of miR-140-5p in a set of 36 archival CRC patient samples of primary tumor tissue and corresponding normal colorectal mucosa. Consistent with previous findings $[13,14]$, we found that miR-140-5p was downregulated in human CRC tissues compared with non-cancerous tissues. Downregulation of miR-140-5p was significantly associated with lymph node metastasis, advanced clinical stage and poorer overall survival. miR-140$5 p$ expressions were also downregulated in different human CRC cell lines. These findings revealed a possible tumor suppressive role for miR-140-5p during CRC development and progression.

Although miR-140-5p is generally regarded as a tumor suppressor in many human malignancies, previous studies have showed a discrepancy for its role in cancer development depending on different cell context $[13,14,26]$. A progressive loss of miR-140-5p has been well demonstrated from normal colorectal mucosa to primary CRC tissues in a study by Zhai et al. [14], with further reduction in liver metastatic tissues. Overexpression of miR-140-5p was reported to inhibit cell proliferation, invasion and cell cycle arrest in both CRC cell lines and cancer stem cell line $[13,14]$. In contrast, Mosakhani and colleague found that miR-140$5 \mathrm{p}$ up-regulation were significantly associated with poorer overall survival in metastatic CRC patients with wild type KRAS/BRAF [26]. It is worth noting that miR-140-5p is up-regulated in colon cancer stem-like cells, despite its downregulation in primary CRC tissue. Expression of miR-140-5p is related to chemoresistance, and blocking it partially sensitizes colon cancer stem-like cells that are resistant to 5-FU treatment [13]. These seemingly contradictory results can be explained by the emerging paradigm that miRNA can be not only cell-type or tissue specific "signatures" for certain normal or cancerous tissues, but also functional or inoperative to certain genes depending on the molecular and cellular context. Thus, one must be cautious in extrapolating the results of interaction between miRNA and its target beyond the research field.

To better understand the role of miR-140-5p in CRC tumorigenesis, we evaluated the effects of miR-140-5p on cell proliferation, apoptosis, migration and invasion. Restoration of miR-140-5p could markedly suppressed cell proliferation and enhance apoptosis of CRC cell lines. Cell migration and invasion are required for tumor cells spreading from the primary site to lymph or blood vessels. Consistent with the clinical findings that miR-140-5p level was inversely correlated with lymph node metastasis and tumor stage, wound healing assays and Transwell assays with Matrigel confirmed the suppressive role of miR-140-5p in cell migration and invasion. Accordingly, miR-140-5p depletion by miR-140-5p inhibitor promoted cell growth and invasion.

It is generally accepted that miRNAs carry out their function by modulating the expression of their target genes. VEGFA is a member of the VEGF family, which has commonly been acknowledged as the most prominent factor to initiate the formation of immature vessels in tumor. Of the six reported subtypes of VEGF, VEGFA increases vascular permeability, degeneration of extracellular matrix and cell aggravation. In CRC, VEGFA signaling-involved neovascularity represents a key mediator of tumor angiogenesis, invasion and dissemination [29]. VEGF levels are known to be increased in CRC, and are associated with an increased ability of the malignancy to spread and with poorer prognosis $[27,28]$. Bevacizumab, a specific anti-VEGF drug that has led to over survival times of about 24 months when combined with standard chemotherapy regimens compared with about 20 months when treated with standard chemotherapy alone [29, 30]. Some VEGFA-targeted miRNAs including miR-203 [31], miR-497 [32], miR-26a [33], and miR-199a-5p [34] have 


\begin{tabular}{|c|c|c|}
\hline Cellular Physiology & Cell Physiol Biochem 2015;37:1123-1133 & \\
\hline and Biochemistry & $\begin{array}{l}\text { DOI: } 10.1159 / 000430237 \\
\text { Published online: September 25, } 2015\end{array}$ & $\begin{array}{l}\text { O } 2015 \text { S. Karger AG, Basel } \\
\text { www.karger.com/cpb }\end{array}$ \\
\hline
\end{tabular}

been reported recently and exhibit an anti-tumor effect. According to several algorithms, we predicted that miR-140-5p may directly target VEGFA. Using a dual-luciferase reporter assay we showed that miR-140-5p directly bound to the 3'UTR of VEGFA, which contains a miR-140-bingding site. Overexpression of miR-140-5p significantly reduced VEGFA mRNA and protein expressions, whereas knockdown of miR-140-5p enhanced VEGFA expression. VEGFA expression had a remarkable inverse correlation with miR-140-5p expression in CRC tissues. Re-expression of VEGFA in miR-140-5p-overexpressed cells reversed the effects of miR-140-5p, suggesting an anti-proliferative role of miR-140-5p attributable largely to VEGFA downregulation.

In conclusion, the present study showed that miR-140-5p is frequently downregulated in CRC tissues and the level of miR-140-5p is closely associated with overall survival of CRC patients. Overexpression of miR-140-5p inhibits cell growth and aggressive behaviors of CRC through directly targeting VEGFA. Our data suggest that the frequently downregulated miR-140-5p leads to the increased expression of VEGFA and in turn contributes to the development and progression of CRC.

\section{Acknowledgements}

This study was supported by the key R\&D Special Fund of Jiangsu Province (BE2015666), China Postdoctoral Science Foundation (2015M571703) and National Science Foundation of China (81500193). The funders had no role in study design, data collection and analysis, decision to publish, or preparation of the manuscript.

\section{Declaration Statement}

The authors report no conflicts of interest.

\section{References}

1 Siegel R, Desantis C, Jemal A: Colorectal cancer statistics, 2014. CA Cancer J Clin 2014;64:104-117.

2 Hegde SR, Sun W, Lynch JP: Systemic and targeted therapy for advanced colon cancer. Expert Rev Gastroenterol Hepatol 2008;2:135-149.

3 Bartel DP: MicroRNAs: genomics, biogenesis, mechanism, and function. Cell 2004;116:281-297.

4 Forman JJ, Legesse-Miller A, Coller HA: A search for conserved sequences in coding regions reveals that the let-7 microRNA targets Dicer within its coding sequence. Proc Natl Acad Sci U S A 2008;105:14879-14884.

5 Ventura A, Jacks T: MicroRNAs and cancer: short RNAs go a long way. Cell 2009;136:586-591.

6 Mendell JT: MicroRNAs: critical regulators of development, cellular physiology and malignancy. Cell Cycle 2005;4:1179-1184.

7 Xuan Y, Yang H, Zhao L, Lau WB, Lau B, Ren N, Hu Y, Yi T, Zhao X, Zhou S, Wei Y: MicroRNAs in colorectal cancer: small molecules with big functions. Cancer Lett 2015;360:89-105.

8 Dong Y, Yu J, Ng SS: MicroRNA dysregulation as a prognostic biomarker in colorectal cancer. Cancer Manag Res 2014;6:405-422.

9 Yang J, Qin S, Yi C, Ma G, Zhu H, Zhou W, Xiong Y, Zhu X, Wang Y, He L, Guo X: MiR-140 is co-expressed with Wwp2-C transcript and activated by Sox 9 to target Sp1 in maintaining the chondrocyte proliferation. FEBS Lett 2011;585:2992-2997.

10 Iorio MV, Visone R, Di Leva G, Donati V, Petrocca F, Casalini P, Taccioli C, Volinia S, Liu CG, Alder H, Calin GA, Menard S, Croce CM: MicroRNA signatures in human ovarian cancer. Cancer Res 2007;67:8699-8707.

11 Yuan Y, Shen Y, Xue L, Fan H: miR-140 suppresses tumor growth and metastasis of non-small cell lung cancer by targeting insulin-like growth factor 1 receptor. PLoS One 2013;8:e73604.

12 Li W, He F: Monocyte to macrophage differentiation-associated (MMD) targeted by miR-140-5p regulates tumor growth in non-small cell lung cancer. Biochem Biophys Res Commun 2014;450:844-850.

13 Song B, Wang Y, Xi Y, Kudo K, Bruheim S, Botchkina GI, Gavin E, Wan Y, Formentini A, Kornmann M, Fodstad O, Ju J: Mechanism of chemoresistance mediated by miR-140 in human osteosarcoma and colon cancer cells. Oncogene 2009;28:4065-4074.

14 Zhai H, Fesler A, Ba Y, Wu S, Ju J: Inhibition of colorectal cancer stem cell survival and invasive potential by hsamiR-140-5p mediated suppression of Smad2 and autophagy. Oncotarget. Oncotarget 2015;6:19735-19746. 


\section{Cellular Physiology Cell Physiol Biochem 2015;37:1123-1133 \begin{tabular}{l|l} 
DOI: 10.1159/000430237 & (C) 2015 S. Karger AG, Basel
\end{tabular} www.karger.com/cpb \\ Zhang et al.: miR-140-5p in CRC}

15 Yang H, Fang F, Chang R, Yang L: MicroRNA-140-5p suppresses tumor growth and metastasis by targeting transforming growth factor beta receptor 1 and fibroblast growth factor 9 in hepatocellular carcinoma. Hepatology 2013;58:205-217.

16 Zhang Y, Eades G, Yao Y, Li Q Zhou Q: Estrogen receptor alpha signaling regulates breast tumorinitiating cells by down-regulating miR-140 which targets the transcription factor SOX2. J Biol Chem 2012;287:41514-41522.

17 Li W, Jiang G, Zhou J, Wang H, Gong Z, Zhang Z, Min K, Zhu H, Tan Y: Down-regulation of miR-140 induces EMT and promotes invasion by targeting Slug in esophageal cancer. Cell Physiol Biochem 2014;34:14661476.

18 Sand M, Skrygan M, Sand D, Georgas D, Hahn SA, Gambichler T, Altmeyer P, Bechara FG: Expression of microRNAs in basal cell carcinoma. Br J Dermatol 2012;167:847-855.

19 Nicolas FE, Pais H, Schwach F, Lindow M, Kauppinen S, Moulton V, Dalmay T: mRNA expression profiling reveals conserved and non-conserved miR-140 targets. RNA Biol 2011;8:607-615.

20 Pais H, Nicolas FE, Soond SM, Swingler TE, Clark IM, Chantry A, Moulton V, Dalmay T: Analyzing mRNA expression identifies Smad3 as a microRNA-140 target regulated only at protein level. RNA 2010;16:489494.

21 Miyaki S, Sato T, Inoue A, Otsuki S, Ito Y, Yokoyama S, Kato Y, Takemoto F, Nakasa T, Yamashita S, Takada S, Lotz MK, Ueno-Kudo H, Asahara H: MicroRNA-140 plays dual roles in both cartilage development and homeostasis. Genes Dev 2010;24:1173-1185.

22 Malzkorn B, Wolter M, Liesenberg F, Grzendowski M, Stuhler K, Meyer HE, Reifenberger G: Identification and functional characterization of microRNAs involved in the malignant progression of gliomas. Brain Pathol 2010;20:539-550.

23 Tardif G, Hum D, Pelletier JP, Duval N, Martel-Pelletier J: Regulation of the IGFBP-5 and MMP-13 genes by the microRNAs miR-140 and miR-27a in human osteoarthritic chondrocytes. BMC Musculoskelet Disord 2009;10:148.

24 Shersher DD, Vercillo MS, Fhied C, Basu S, Rouhi O, Mahon B, Coon JS, Warren WH, Faber LP, Hong E, Bonomi P, Liptay MJ, Borgia JA: Biomarkers of the insulin-like growth factor pathway predict progression and outcome in lung cancer. Ann Thorac Surg 2011;92:1805-1811; discussion 1811.

25 Ahn BY, Elwi AN, Lee B, Trinh DL, Klimowicz AC, Yau A, Chan JA, Magliocco A, Kim SW: Genetic screen identifies insulin-like growth factor binding protein 5 as a modulator of tamoxifen resistance in breast cancer. Cancer Res 2010;70:3013-3019.

26 Mosakhani N, Lahti L, Borze I, Karjalainen-Lindsberg ML, Sundstrom J, Ristamaki R, Osterlund P, Knuutila S, Sarhadi VK: MicroRNA profiling predicts survival in anti-EGFR treated chemorefractory metastatic colorectal cancer patients with wild-type KRAS and BRAF. Cancer Genet 2012;205:545-551.

27 Ferroni P, Spila A, Martini F, D'Alessandro R, Mariotti S, Del Monte G, Graziano P, Buonomo O, Guadagni F, Roselli M: Prognostic value of vascular endothelial growth factor tumor tissue content of colorectal cancer. Oncology 2005;69:145-153.

28 Des Guetz G, Uzzan B, Nicolas P, Cucherat M, Morere JF, Benamouzig R, Breau JL, Perret GY: Microvessel density and VEGF expression are prognostic factors in colorectal cancer. Meta-analysis of the literature. $\mathrm{Br}$ J Cancer 2006;94:1823-1832.

29 Grothey A, Galanis E: Targeting angiogenesis: progress with anti-VEGF treatment with large molecules. Nat Rev Clin Oncol 2009;6:507-518.

30 Thornton AD, Ravn P, Winslet M, Chester K: Angiogenesis inhibition with bevacizumab and the surgical management of colorectal cancer. Br J Surg 2006;93:1456-1463.

31 Zhu X, Er K, Mao C, Yan Q, Xu H, Zhang Y, Zhu J, Cui F, Zhao W, Shi H: miR-203 suppresses tumor growth and angiogenesis by targeting VEGFA in cervical cancer. Cell Physiol Biochem 2013;32:64-73.

32 Wang W, Ren F, Wu Q, Jiang D, Li H, Shi H: MicroRNA-497 suppresses angiogenesis by targeting vascular endothelial growth factor A through the PI3K/AKT and MAPK/ERK pathways in ovarian cancer. Oncol Rep 2014;32:2127-2133.

33 Chai ZT, Kong J, Zhu XD, Zhang YY, Lu L, Zhou JM, Wang LR, Zhang KZ, Zhang QB, Ao JY, Wang M, Wu WZ, Wang L, Tang ZY, Sun HC: MicroRNA-26a inhibits angiogenesis by down-regulating VEGFA through the PIK3C2alpha/Akt/HIF-1alpha pathway in hepatocellular carcinoma. PLoS One 2013;8:e77957.

34 Hsu CY, Hsieh TH, Tsai CF, Tsai HP, Chen HS, Chang Y, Chuang HY, Lee JN, Hsu YL, Tsai EM: miRNA-199a$5 p$ regulates VEGFA in endometrial mesenchymal stem cells and contributes to the pathogenesis of endometriosis. J Pathol 2014;232:330-343. 